NASA Technical Memorandum102273

\title{
Temporally and Spatially Resolved Flow in a Two-Stage Axial Compressor, Part 2 - Computational Assessment
}

Karen L. Gundy-Burlet, Man Mohan Rai, R. Charles Stauter, and Robert P. Dring

January 1990

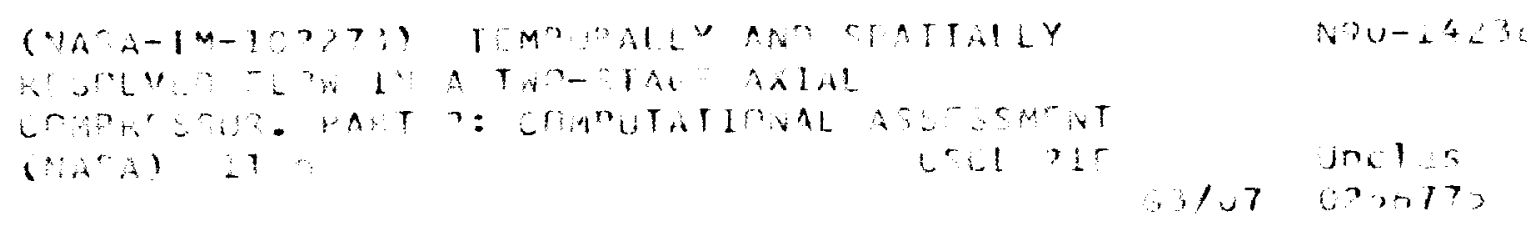




\section{,}




\section{Temporally and Spatially Resolved Flow in a Two-Stage Axial Compressor, Part 2 - Computational Assessment}

Karen L. Gundy-Burlet, Man Mohan Rai, Ames Research Center, Moffett Field, California

R. Charles Stauter and Robert P. Dring,

United Technologies Research Center, East Hartford, Connecticut

January 1990

\section{N/S}

National Aeronautics and

Space Administration

Ames Research Center

Moffett Field, California 94035 



\title{
TEMPORALLY AND SPATIALLY RESOLVED FLOW IN A TWO-STAGE AXIAL COMPRESSOR, PART 2 - COMPUTATIONAL ASSESSMENT
}

\author{
Karen L. Gundy-Burlet and Man Mohan Rai \\ NASA Ames Research Center \\ Moffett Field, CA 94035-1000 \\ and \\ R. Charles Stauter and Robert P. Dring \\ United Technologies Research Center \\ East Hartford, CT 06108
}

\begin{abstract}
Fluid dynamics of turbomachines are complicated because of aerodynamic interactions between rotors and stators. It is necessary to understand the aerodynamics associated with these interactions in order to design turbomachines that are both light and compact as well as reliable and efficient. The current study uses an unsteady, thin-layer Navier-Stokes zonal approach to investigate the unsteady aerodynamics of a multistage compressor. Relative motion between rotors and stators is made possible by the use of systems of patched and overlaid grids. Results have been computed for a $2 \frac{1}{2}$-stage compressor configuration. The numerical data compare well with experimental data for surface pressures and wakes. In addition, the effect of grid refinement on the solution is studied.

\section{Nomenclature}

$\begin{array}{ll}C_{p} & \text { Pressure coefficient, } C_{p}=\frac{P-P_{T_{i}}}{\frac{1}{2} \rho_{i} U_{m}^{2}} \\ C_{x} & \text { Inlet axial velocity } \\ \mathrm{F} & \text { Airfoil force per unit span normalized by } Q_{U_{m}} \\ & \text { and } \tau \\ \mathrm{P} & \text { Pressure } \\ P_{T_{i}} & \text { Compressor inlet absolute Total Pressure } \\ Q_{U_{m}} & \text { Dynamic pressure based on } U_{m} \\ \mathrm{~T} & \text { Time normalized by airfoil passing period } \\ \mathrm{t} & \text { Tangential distance normalized by } \tau \\ U_{m} & \text { Wheel speed at midspan } \\ \mathrm{W} & \text { Relative flow speed } \\ \mathrm{x} & \text { Axial distance } \\ \rho_{i} & \text { Compressor inlet density } \\ \tau & \text { Airfoil pitch } \\ \phi & \text { Flow coefficient, } \phi=C_{x} / U_{m}\end{array}$
\end{abstract}

\author{
Subscripts \\ t Tangential component \\ $x \quad$ Axial component
}

\section{Introduction}

Simulations of flows within turbomachines are challenging because of the complicated rotating geometries and unsteady flow structures. Designers are often required to place airfoils close together to minimize engine weight and size. This leads to aerodynamic interactions between rotor and stator airfoils. Part of the interaction is due to the inviscid potential effect between closely spaced rotor and stator airfoils. Viscous effects also contribute to the unsteady interactions. The angle of attack of an airfoil can vary widely as it passes through the wake of an upstream airfoil, because of the velocity deficit in the wake. Unsteady variations in angle of attack coupled with local adverse pressure gradients can cause unsteady separation. In addition, trailing-edge vortex shedding can increase the unsteadiness of the system. Simulation of the unsteady flow in a turbomachine can lead to an understanding of the aerodynamic interactions that occur.

Most methods used today to simulate flows in turbomachines are for either cascade flows or time-averaged flows through several airfoil rows. Simulations by Davis et al. (1988), Choi and Knight (1988), Subramanian et al. (1986) and Weinberg et al. (1986) are representative of these methods, but by no means make an exhaustive list of the methods available. Perturbation techniques can be applied to these simulations for an estimate of the unsteadiness for weak interactions. Distorting-grid techniques, described by Gibeling et al. (1986), can be used to investigate flows in which relative motion exists between rotors and stators. In these techniques, a single grid is wrapped 


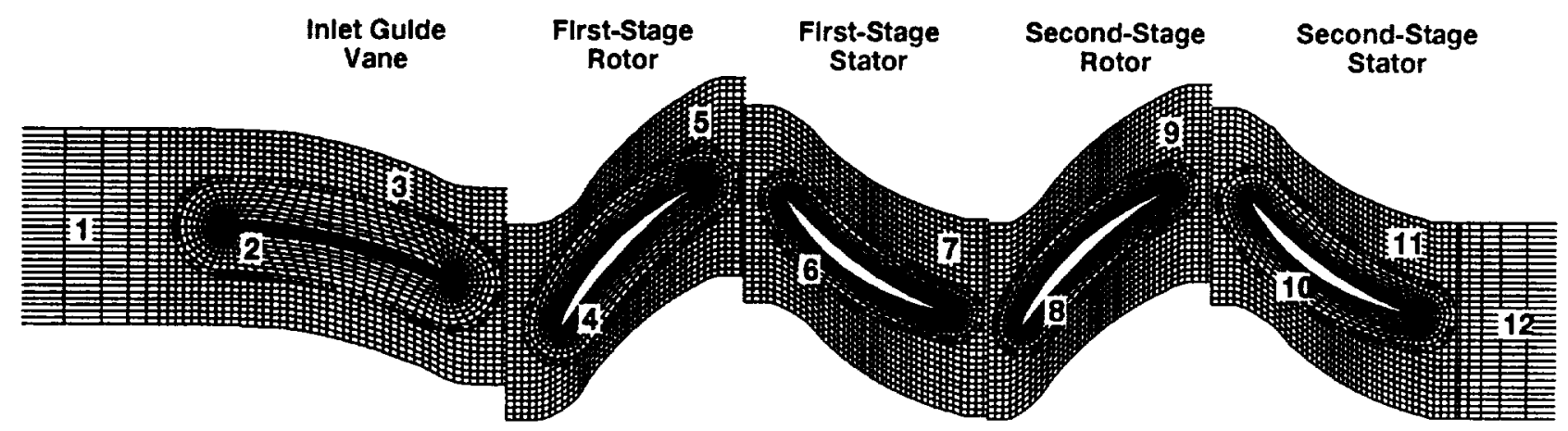

Fig. 1. Zonal grid system.

about both airfoils and sheared as the aiffoils move relative to one another. When the distortion in the grid exceeds a certain level, the solution is interpolated onto a new, undistorted grid. However, this method may produce inaccurate solutions because of the grid distortion if the airfoils are too closely spaced.

For this reason, Rai (1987) and Rai and Madavan (1988) used a system of patched and overlaid grids to compute the rotor-stator interaction problem in a single-stage turbine. Body-fitted " $O$ " grids with fine-grid spacing at the airfoil surfaces were used to capture viscous effects near the airfoil surface. These " $O$ " grids were overlaid on sheared Cartesian "H" grids, which were allowed to slip past each other to simulate the interaction problem. The ROTOR2 code resulted from these efforts to simulate rotor-stator interaction for single-stage turbine configurations. The STAGE-2 code, used in the present study, is an extension of ROTOR-2 for multistage turbomachines. Results from STAGE-2 were compared with experimental data for both a multistage compressor and a single-stage turbine in GundyBurlet et al. (1989).

STAGE-2 is currently being used to further investigate the flow in the multistage compressor. The $2 \frac{1}{2}$-compressor geometry was chosen for the present analysis because a large body of experimental data is evailable for the multistage compressor. Much of the experimental data is summarized and tabulated in Dring and Joslyn (1985). This compressor is also part of the AGARD (1989) collection of test cases for computation of internal flows in aero-engine components. In addition, both steady and unsteady laser doppler velocimetry (LDV) data have been taken in the second stage of the compressor. These data are presented by Stauter, Dring, and Carta (1990) in Part 1 of this paper. Comparisons, in the present report, of STAGE-2 calculations with experimental data include time-averaged pressures and wake velocity profiles. The effect of grid refinement on the solution is studied, and instantaneous entropy contours for the $2 \frac{1}{2}$-stage compressor are presented. The computed results are in good agreement with experimental data.

\section{Approach}

The current work is an extension of an algorithm developed by Rai and is discussed in detail in Raj and Chakra- varthy (1986). The algorithm is discussed in brief here. The flow field is divided into two basic types of zones. The thin-layer Navier-Stokes equations are solved in inner zones near the airfoil where viscous effects are important. The Euler equations are used in outer zones where viscous effects are weak. These equations are cast in the strong conservative form. A fully implicit finite difference method is used to advance the solution of the nonlinear equations in time. At each time step, four Newton-Raphson iterations are used to reduce the factorization and linearization errors by an order of magnitude. The convective terms are evaluated using a third-order-accurate upwind-biased Osher scheme, and the viscous terms are evaluated using second-order central differences. The Baldwin-Lomax (1978) turbulence model is used to compute the turbulent eddy viscosity. Details of the turbulence model, zonal and natural boundary conditions, grid configuration, bookkeeping system, and database management systems are discussed in Gundy-Burlet et al. (1989).

\section{Grid System}

The grid system for the $2 \frac{1}{2}$-stage compressor geometry is shown in Fig. 1. The experimental compressor geometry consists of an inlet guide vane (IGV) followed by two nearly identical stages. The only difference between the stages is that the first-stage rotor is closed $3^{\circ}$ relative to the second-stage rotor. The only deviation from the original design was that the axial gaps between the rotors and stators were increased slightly to permit the incorporation of a radial-circumferential traverse system. All airfoils are defined by NACA 65 -series sections with circular-arc mean camber lines. There are 44 airfoils in each row. The hub and casing are at a constant radius along the axis of the compressor. These features make the compressor ideal for computational modeling.

The computation was performed using the midspan experimental geometry. It was assumed that the flow is periodic from airfoil to airfoil in the circumferential direction, so the flow through only one airfoil-to-airfoil passage was computed. In addition, the experimental IGV geometry was unavailable at the time this calculation was initiated, so a $0.8 \%$-thick parabolic arc airfoil was used. These assumptions are not expected to significantly affect the comparison between the computation and the experiment. 


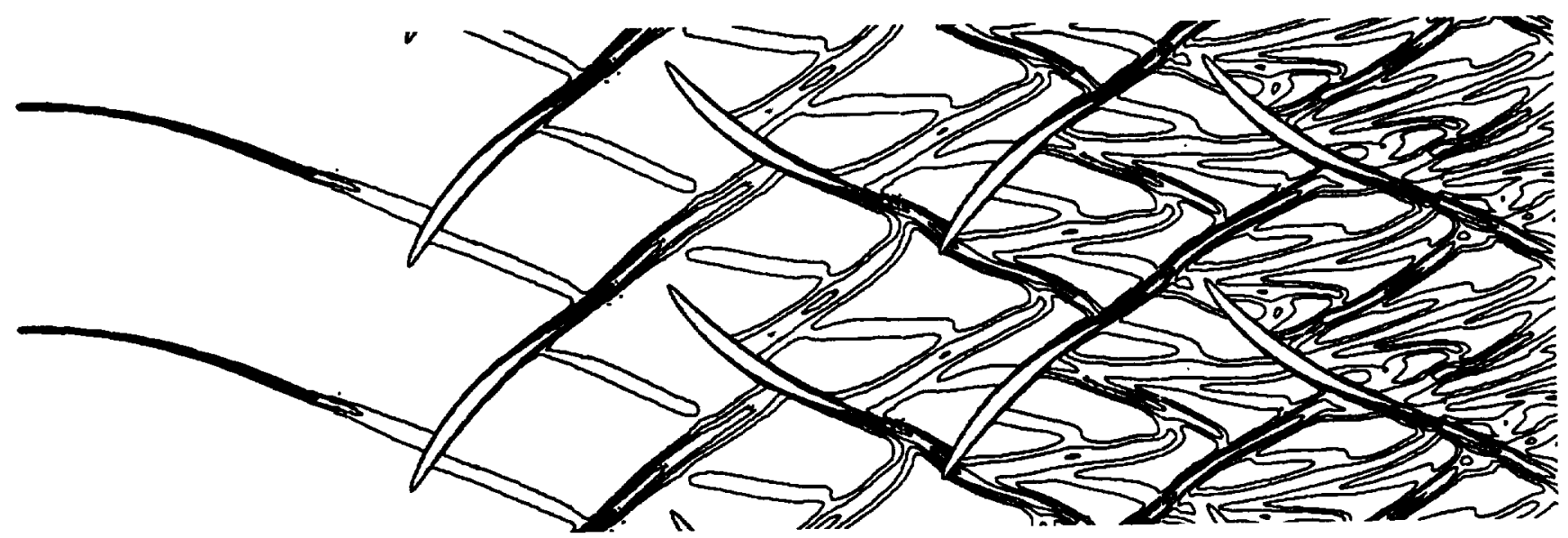

Fig. 2. Instantaneous entropy contours.

Two types of grids are used to discretize the flow about each of the airfoils, as seen in Fig. 1. An inner " $O$ " grid, generated using an elliptical method, is used to resolve the viscous effects near the airfoil. The " $O$ " grid is then overlaid on an algebraically generated, sheared Cartesian outer grid. The outer grids are allowed to slip past each other to model the relative motion between rotors and stators. Using this system, 12 grids are required to fully discretize the flow field through the $2 \frac{1}{2}$-stage compressor.

Two different grids were used to study the effect of grid refinement on the solution. Every other point in the coarse grid is displayed in Fig. 1. The inner grids have $151 \times 31$ grid points each, and the outer grids have $86 \times 61$ grid points each. The inlet and exit grids have $28 \times 61$ and $30 \times 61$ grid points each, respectively. The total number of points in all 12 zones of the coarse grid is 53,173 .

Experimental data exists only for the second stage of the compressor. In order to reduce the computational time required for a grid refinement study, grid refinement was done only in the second stage of the compressor. The second-stage inner grids were refined to have $301 \times 61$ points each while the second-stage outer grids were refined to have $171 \times 121$ grid points each. The exit-grid size was increased to $59 \times 121$. Thus, the fine-grid cells are one quarter the area of the coarse-grid cells. The total number of points for the fine-grid system was 116,732 .

\section{Results}

Results for the $2 \frac{1}{2}$-stage compressor configuration shown in Fig. 1 are presented in this section. For the 53,000 -point coarse grid used in this study, 500 time steps are used for each blade-passing time. Each time step requires 3.9 CPU seconds on a Cray YMP. Time-averaged quantities converge within 10 blade passings, but the presence of multiple stages increases the time required to achieve a time-periodic solution, as compared to a singlestage system. Acoustic signals must travel a larger distance to exit from a multistage system. In addition, these signals reflect off airfoils and the reflections take time to exit the system. Depending on the level of convergence required of the time-periodic solution, between 5 and 40 hours of CPU time are needed for the calculations.
Several items must be taken into consideration when comparing these results with the experimental data. The experiment is inherently three-dimensional, while the computations are two-dimensional. Hub-corner separation, tipleakage effects, and end-wall boundary-layer growth are not modeled. In the experiment, the end-wall boundary-layer growth generates a stream-tube contraction that increases the axial velocity at midspan along the axis of the compressor; the midspan exit axial velocity is increased to $110 \%$ of the midspan inlet velocity. This increase is not reflected in the calculation because the stream-tube contraction is currently not modeled.

The calculation was performed at an inlet Mach number of 0.07 and a Reynolds number of 100,000 per inch based on inlet conditions. These values were set to match the experimental operating conditions. The experimental flow coefficient is $\phi=0.51$. The exit static-pressure coefficient for the experiment is $C_{p}=1.11$. It is not possible to match the computational values with both the experimental pressure rise and the experimental flow coefficient because the stream-tube contraction is not modeled.

The first comparison typically made between calculation and experiment is of the time-averaged pressures on the rotors and stators. As data is available only for the second stage of the compressor, setting the exit pressure equal to the experimental value and allowing the flow coefficient to float provide a reasonable comparison between computation and experiment. The mass flow rate in the calculation then adjusts itself to the pressure rise specified. Only the Riemann invariants (functions of velocity and speed of sound), total pressure, and flow angle are specified at the inlet, so the final time-averaged values of velocity and static pressure may vary from the initial condition. The flow coefficient in the calculation increased to $\phi=0.524$ as the periodic steady state was reached. The flow coefficient for the experiment was $\phi=0.51$.

In order to demonstrate the complexity of the flow field, instantaneous entropy contours are shown in Fig. 2. Entropy was chosen to display the viscous nature of the flow because, unlike total pressure, entropy is invariant with reference frame. These results were computed using refined grids in the second stage of the compressor. The entropy 
contours illustrate the depth and width of the wake for the inlet guide vane as well as for the rotors and stators. The wakes generated in the second stage of the compressor are better resolved than the wakes generated in the first stage of the compressor. Although it is difficult to see this in Fig. 2 , the difference in resolution of the wakes becomes clear in subsequent figures (in particular, Fig. 5). The numerical dissipation associated with the coarse-grid resolution of the first stage of the compressor causes the wakes to diffuse more rapidly than they would have with a fine grid. Because the wakes from the first stage of the compressor are more diffuse, the general level of unsteadiness seen by the second stage of the compressor (especially the second-stage rotor) is expected to be underpredicted. All grids would have to be refined to get a more accurate estimate of the amplitude of the unsteady behavior in the compressor.

The flow field in the second stage of the compressor is complicated by the accumulation of the convected wakes. Wakes from the first-stage rotor and stator penetrate into the second stage of the compressor, despite the lack of grid resolution in the first stage. Both wake/wake interactions and wake/airfoil interactions occur in the flow field. The impact of the difference in convection rate from the suction side of an airfoil to the pressure side of an airfoil is also evident, particularly on the second-stage rotor. The circulation around the airfoils produces a streamwise displacement of the wakes as they are convected along the suction and pressure surfaces. The circulation also causes a rotation of the chopped-wake segments as they are convected through the airfoil-to-airfoil passages. It is clear from Fig. 2 that wakes can be convected many chords downstream to interact with other airfoils. This causes unsteady variations in flow variables in the later stages of the compressor to be more complex than in the early stages.

As the downstream airfoils move through the wakes of the upstream aiffoil rows, the wake/airfoil interaction causes the surface pressures to fluctuate. The airfoil surface pressures also fluctuate because of interactions with the potential flow fields of the upstream and downstream airfoil rows. The pressure distributions for the second-stage rotor and stator are presented in Fig. 3 in terms of the timeaveraged surface pressures. The shaded band indicates the temporal maximum and miminum over a cycle caused by the fluctuations mentioned above. The time-averaged static pressure is arrived at by averaging the instantaneous static pressures over one cycle. A cycle is defined as the time it takes for the rotor to move from its position relative to one stator to the corresponding position with respect to the next stator. Pressure is plotted against axial position in the compressor. The axial distance between the rotor and stator results is the actual axial gap.

The symbols in Fig. 3 represent measured data for this compressor that had been reported previously by Joslyn and Dring (1989a). The data was acquired using long lengths of instrumentation tubing between the rotor and stator surface pressure taps and the rotating and stationary frame transducers, respectively. Thus, the measurements are presumed to be indicative of the time-averaged surface static pressure.

The agreement between the calculation and the exper- imental data is good. The small differences between them at the leading and trailing edges are due primarily to the mismatch in axial velocity. Although the flow coefficient in the experiment was 0.51 when averaged from hub to tip, a stream-tube contraction caused the flow coefficient at the midspan to be 0.54 at the inlet to the second stage.

The band in Fig. 3 indicating the temporal maximum and minimum of the local surface static pressure on the rotor and stator is nearly uniform in width around both sides of both airfoils. The increase in this unsteady amplitude near the leading edges may be related to the wakes of the upstream airfoil. However, it may also be related to the large gradient in the time-averaged pressure at the leading edge. The amplitude of the unsteady static pressure is approximately $10 \%$ of the airfoil loading. The pressure amplitude is also evident in the pressure-force results presented in Fig. 4 and discussed below. Both the rotor and stator airfoils show a pressure spike at the trailing edge. The trailing-edge spikes in this fine-grid calculation are onethird the size of the trailing-edge spikes in the coarse-grid calculation; this shows that the spikes are strongly dependent on grid resolution.

Close inspection of computed flow velocities at one instant in time near the second-stage stator shows the presence of a thin separation bubble from about $80 \%$ chord to the stator trailing edge. The separation bubble became apparent only under very high magnification of the results. It is undetectable at the scale of the results presented in Fig. 2 and does not appear to strongly affect the comparison with experment in Fig. 3. The temporal behavior of the separation bubble has not yet been examined.

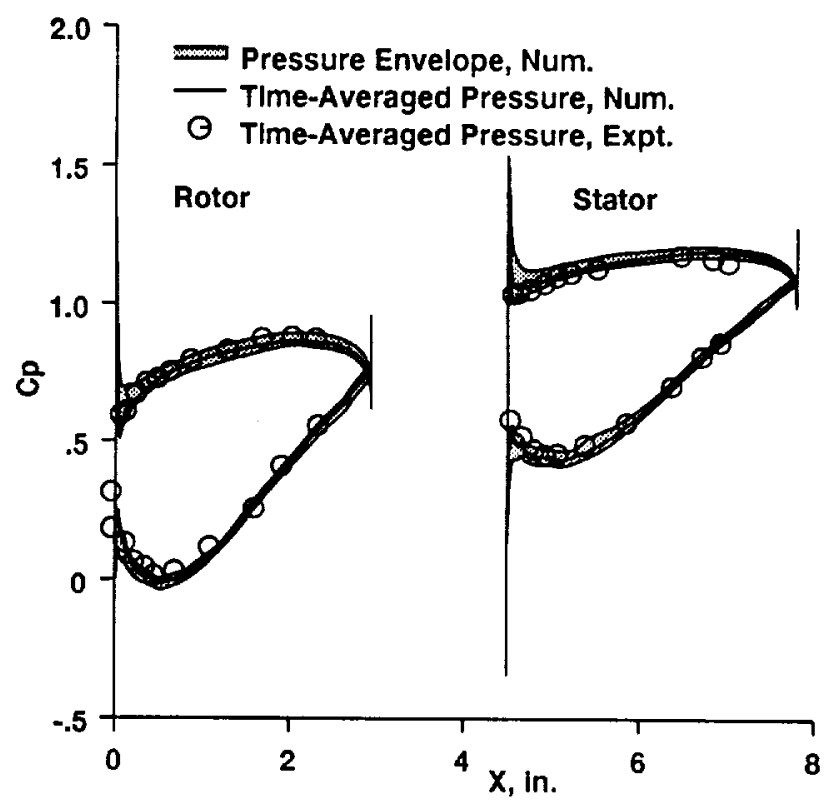

Fig. 3. Surface pressures in the second stage of the compressor. 
The existence of the separation bubble contrasts with previous flow visualization of the airfoils, which had indicated that at midspan both the rotor and stator suctionsurface boundary layers are attached all the way to the trailing edge (Joslyn and Dring, 1989b). These two results are reconciled as follows. The turbulence model used in the present calculation is the fully turbulent Baldwin and Lomax (1978) model; Joslyn and Dring (1989b) showed that if the boundary layers were fully turbulent, both the rotor and stator would experience boundary layer separation at about the $80 \%$ chord location. They also demonstrated that the boundary layers had to be transitional to remain attached to the trailing edge. Transitional modeling was beyond the scope of the present calculation.

In spite of the fact that the separation bubble on the stator did not seem to have any major impact on the pressure distribution, its presence should be kept in mind throughout the remainder of this assessment.

The instantaneous surface static pressures were integrated around the perimeter of the rotor and stator to produce the periodic variation of the pressure-force vector on each airfoil for both a coarse-grid and a fine-grid calculation. A similar integration for the skin-friction force has not been carried out yet. The instantaneous axial and circumferential components of the force are plotted as a polar curve for the second-stage rotor in Fig. $4 a$ and for the second-stage stator in Fig. $4 \mathrm{~b}$. The squares indicate the time-averaged values of the forces, computed with the fine grid. The direction of the time-averaged force and the normal to the stagger angle of each airfoil are included in the plots as reference.

These polar plots provide a good measure of the periodic convergence of the solution. At the periodic steady state, the force polar plot for one cycle should lay on top of the curve for the previous cycle. The coarse-grid results shown here are converged to this criterion. The fine-grid results show small variations from cycle to cycle, but are considered close to convergence. A time-accuracy study has been carried out for the coarse grid. Solutions were carried out at time steps of 500, 1000 and 2000 steps per cycle. Although not shown here, the force polar plots for each of these time steps compared well with those for the other time steps. This indicates that the periodic solution is independent of the time steps used in this study.

The coarse- and fine-grid solutions are qualitatively similar for the second-stage rotor shown in Fig. 4a. Because only the grids in the second stage of the compressor were refined for the fine-grid calculation, the wakes for the airfoils upstream of the second-stage rotor are computed on identical grids for both the fine and coarse grids. There are larger differences between the coarse- and fine-grid solutions for the second-stage stator shown in Fig. $4 \mathrm{~b}$, because of the difference in resolution of the second-stage rotor wake. The amplitude of the fine-grid force on the second-stage stator is approximately $40 \%$ larger than the amplitude of the coarse-grid force.

The unsteady forces are dominated by the variation in the lift component of the force. For brevity, "lift" is defined as the component of force normal to the stagger angle and "drag" as the component of force parallel to the stagger angle. The magnitude of the unsteady force is typically $10 \%$ of the time average for the relatively large rotor/stator axial gaps found in this compressor. These axial gaps are

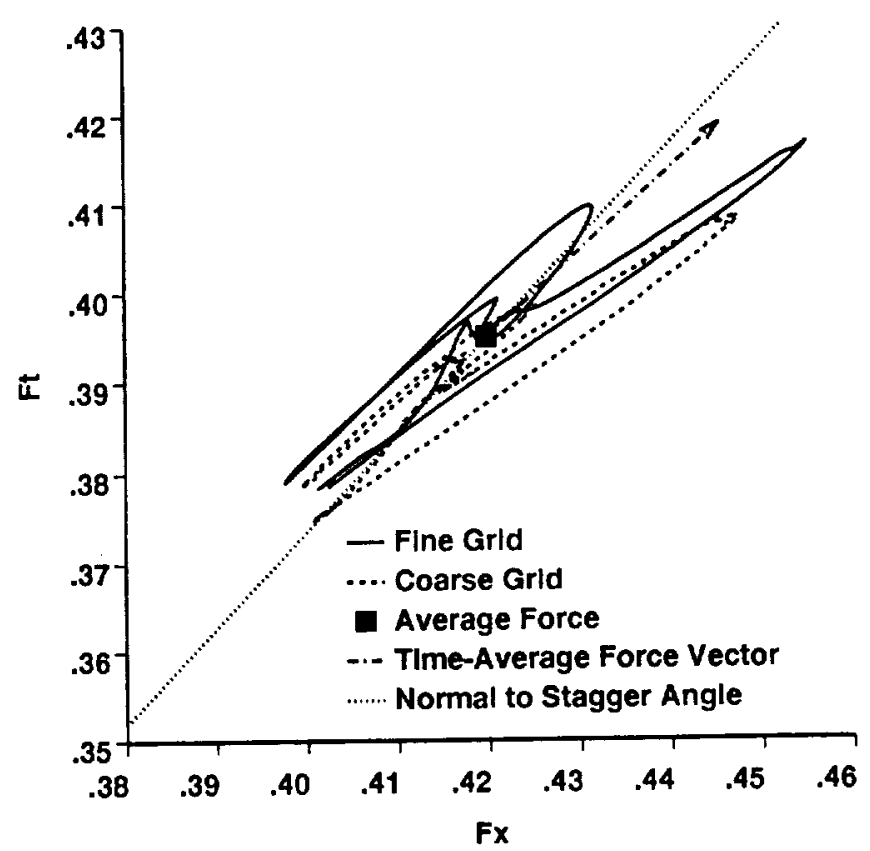

Fig. 4a. Force polar plot for the second stage rotor.

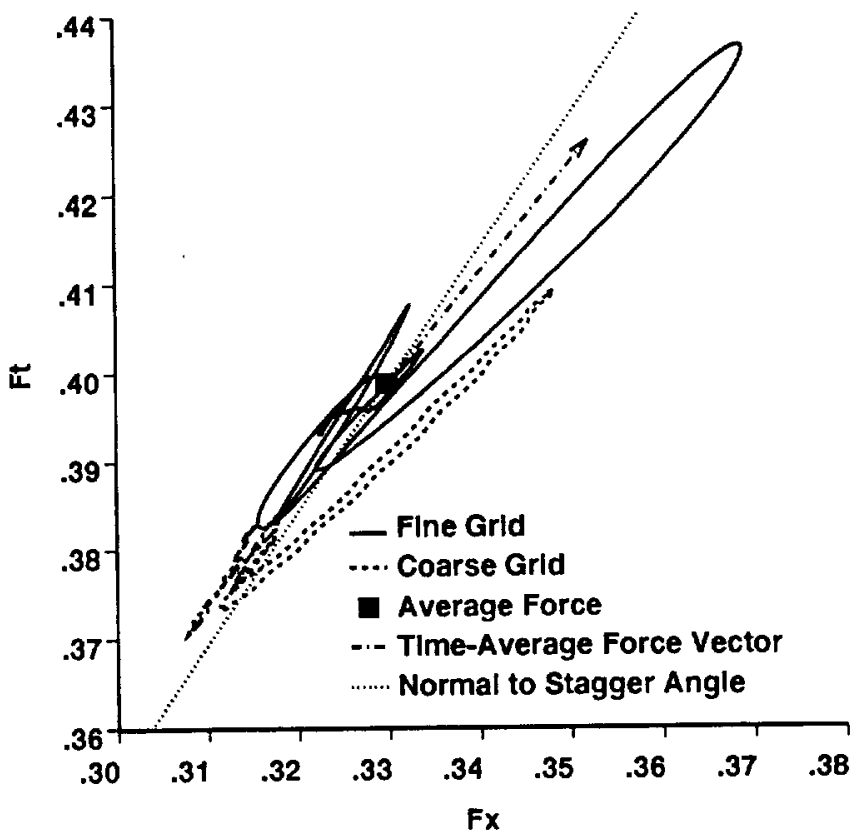

Fig. 4b. Force polar plot for the second stage stator. 
twice as large as those found in a typical modern engine.

The unsteadiness is largely contained in the lift component of the force. Figs. $4 \mathrm{c}$ and $4 \mathrm{~d}$ show the lift and drag components of the force plotted against time for the second-stage rotor and stator, respectively. The curves vary smoothly, indicating that the unsteadiness in the force is not due to a narrow wake, but is distributed more uniformly, as one might expect for a potential interaction. Had the axial gaps between airfoil rows been smaller, the wake contribution might have been more pronounced.

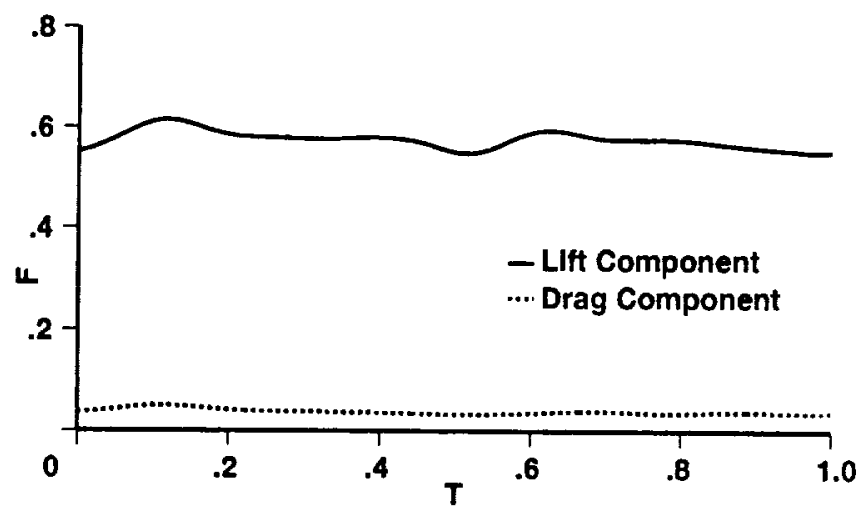

Fig. 4c. Time variation of forces on the second-stage rotor.

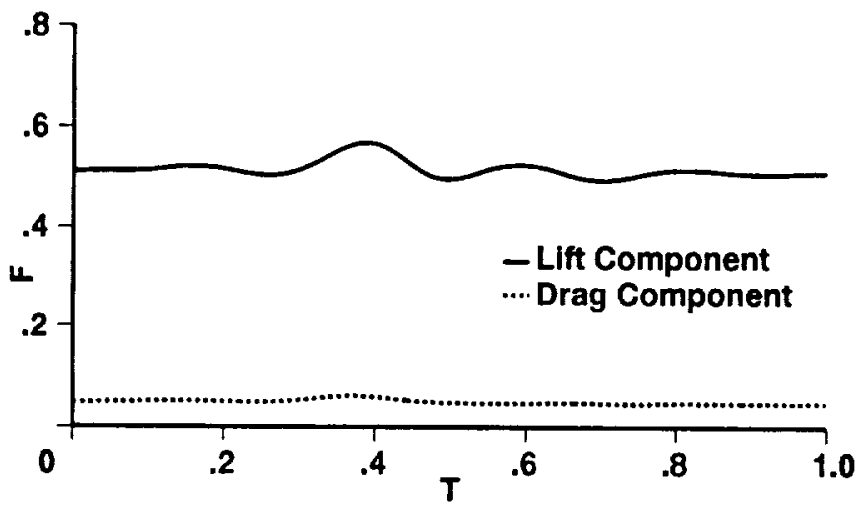

Fig. 4d. Time variation of forces on the second-stage stator.

The effect of grid refinement on wake profiles can be seen in Fig. 5. Time-averaged relative velocities for the coarse grid, fine grid, and experiment (Stauter, et al. 1990) are plotted against tangential displacement from the rotor trailing edge. This comparison is at an axial location $26 \%$ of the rotor axial chord downstream of the second-stage rotor trailing edge. The fine-grid calculation is in good agreement with the experimental data in terms of wake depth, width, and location. The wake depths for the coarse-grid results are shallower than for the experimental data and are visibly displaced. The fine-grid and experimental results have a displacement approximately $50 \%$ greater than those of the coarse grid. This is caused by the different computed rotor exit-flow angles which resulted from the difference in airfoil loading between the coarse- and fine-grid solutions seen in
Fig. 4a; these results also suggest that solutions should be computed with several different grid densities in order to determine the asymptotic behavior of the solution with grid refinement. The remainder of the wake profile comparisons will be between the measured data (Stauter, et al. 1990) and the fine-grid results.

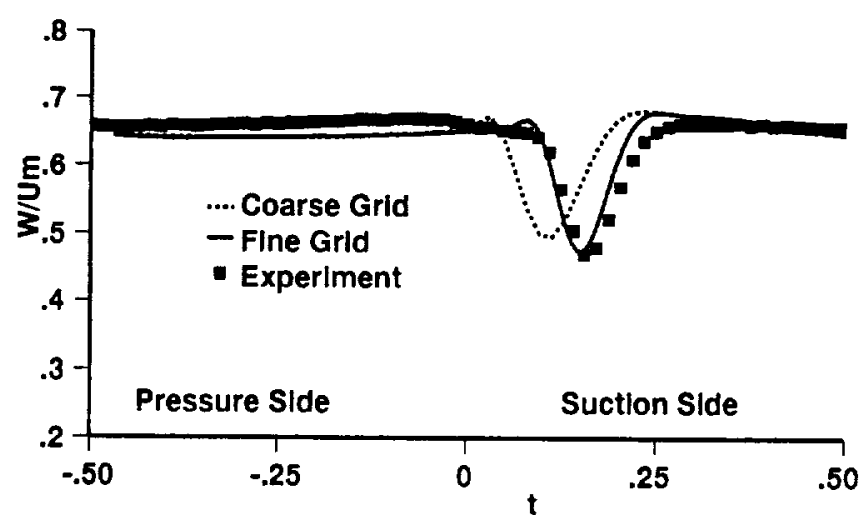

Fig. 5. Wake profiles $26 \%$ behind rotor for fine grid, coarse grid, and experiment.

A comparison of the fine-grid and the experimental data is made at axial locations nominally $2 \%, 8 \%, 15 \%$, and $26 \%$ aft of the rotor trailing edge (Figs. 6a-6d). Both the computed and the measured results are presented in terms of their temporal maximum, temporal minimum, and time average. The temporal fluctuation is caused by the interaction with the upstream stator's wake (see Fig. 2), as well as by the potential field of the downstream stator.

Generally, at each axial location, the agreement is good between the measured and computed time-averaged results for wake depth, width, and tangential location. The greatest differences between the measured and computed results are in the regions between wakes, where there are systematic (albeit small) differences. This can be seen most clearly on the suction-surface side of the wake, where the wake merges with the "free stream". At all axial locations, the prediction at this tangential location is typically $5 \%$ greater than the measured results. These differences are probably related to the nature of the mixing of the wakes, the lack of resolution of the upstream wakes, and the inability to model three-dimensional effects with a two-dimensional calculation.

Flow-speed fluctuations are given, in Figs. 6a-6d, by the width of the band between the instantaneous maximum and minimum relative velocities. The agreement between the measured and computed fluctuations is reasonably good. While the maximum-to-minimum band width of the computation is generally smaller than that of the experiment, many of the features have been captured in the computation, most notably the increase in band width with distance aft of the rotor. As pointed out by Stauter et al. (1990), this increase is due to the potential field of the downstream stator. 


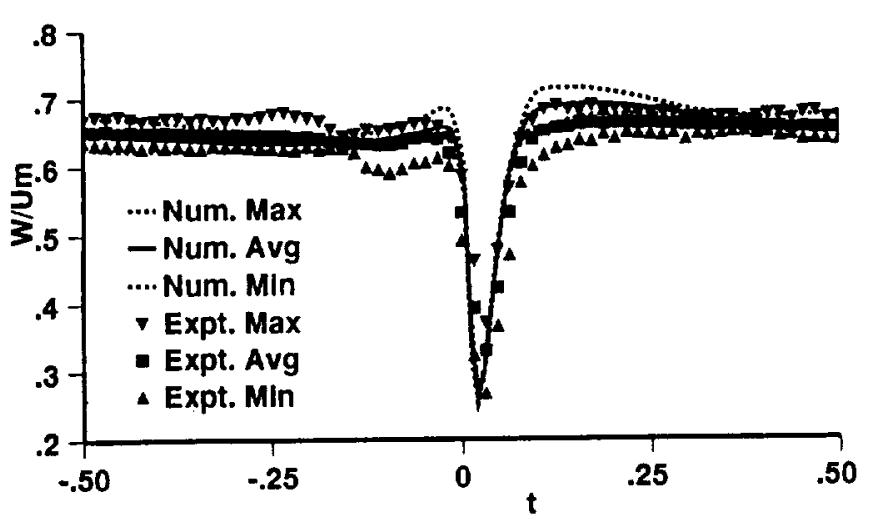

Fig. 6a. Wake profiles $2 \%$ aft of the second-stage rotor.

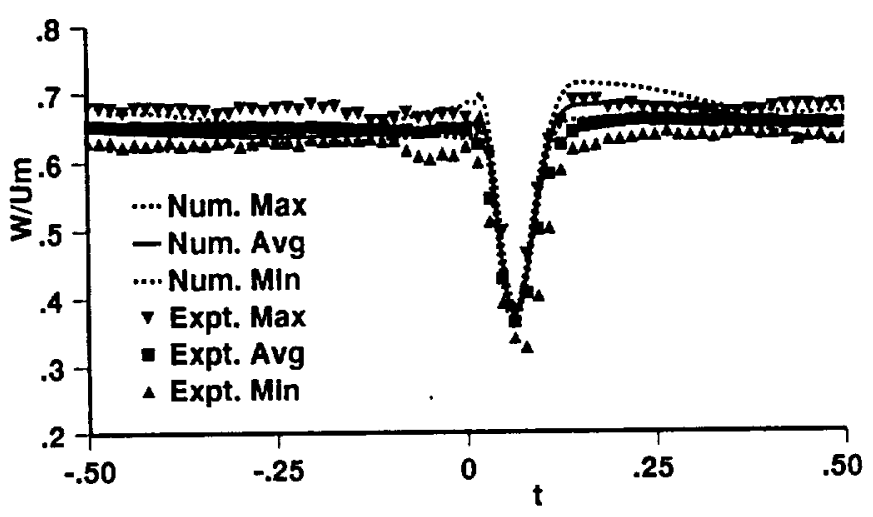

Fig. 6b. Wake profiles $8 \%$ aft of the second-stage rotor.

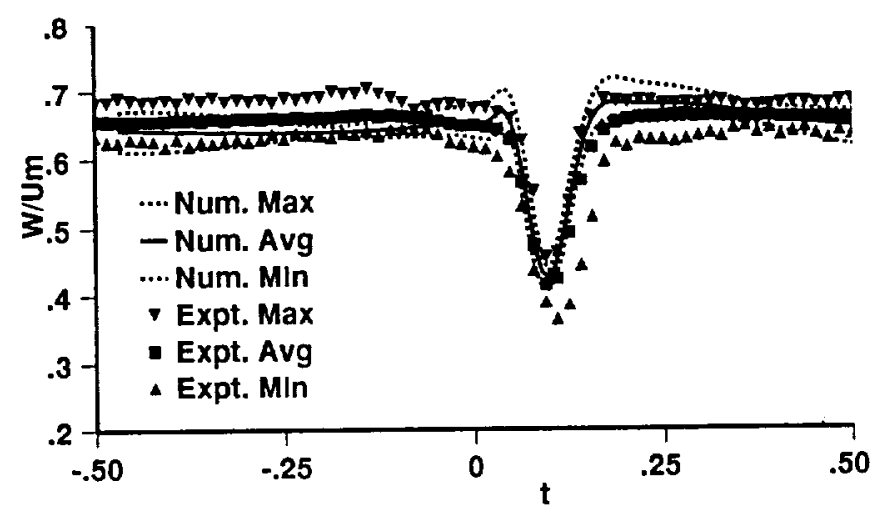

Fig. 6c. Wake profiles $15 \%$ aft of the second-stage rotor.

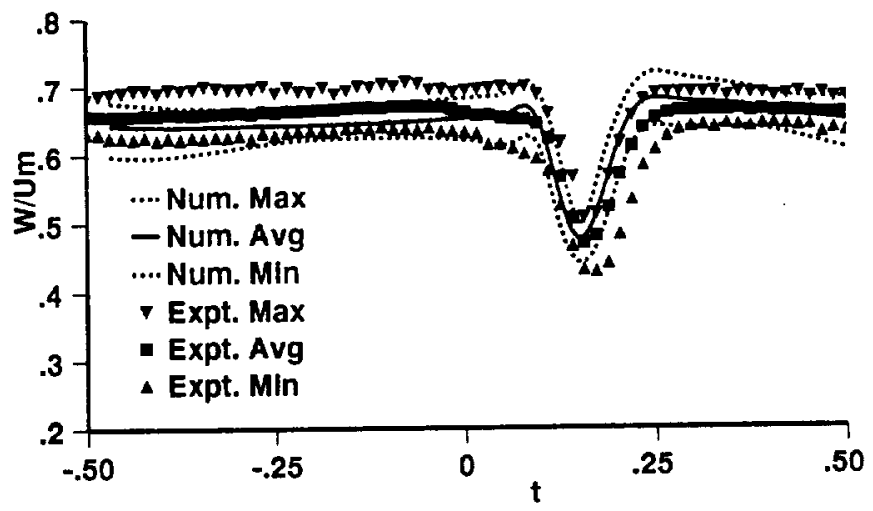

Fig. 6d. Wake profiles $26 \%$ aft of the second-stage rotor.

\section{Conclusions}

A third-order-accurate upwind-biased Navier-Stokes zonal code (STAGE-2) has been developed to compute flows in multistage turbomachines. Systems of overlaid and patched grids are used to resolve viscous effects near airfoils allowing the rotor/stator interaction problem to be modeled. Flexible database and bookkeeping systems are used to allow flows to be computed in turbomachines with any number of stages.

Results from STAGE-2 compared well with experimental data for both surface pressures and wake profiles. Grid refinement substantially improved the agreement with experiment for the wake profiles, although static pressures were relatively insensitive to grid refinement. The temporal variation in airfoil force was $10 \%$ of the time-averaged force for the relatively large axial gaps found in this compressor. Strong viscous interactions were demonstrated by the use of entropy contours. The flow within the second stage of the compressor was highly complex, indicating the importance of unsteady-flow analysis in a multistage turbomachine.

With smaller axial gaps between the rotors and stators of modern engines, it can be anticipated that the interactions in general will be stronger and the unsteady forces in particular will be much larger than those for the compressor studied here.

\section{References}

AGARD, 1989, "Test Cases for Computation of Internal Flows in Aero Engine Components," AGARD Propulsion and Energetics Panel, Working Group 18, AGARDAR-275.

Baldwin, B. S. and Lomax, H., 1978, "Thin Layer Approximation and Algebraic Model for Separated Turbulent Flow," AIAA Paper 78-257, Jan.

Choi, D. and Knight, C. J., 1988, "Computation of 3D Viscous Cascade Flows," AIAA Paper 88-0363, Reno, Nev., Jan.

Dring, R. P., and Joslyn, H. D., 1985, "An Assessment of Single- and Multi-Stage Compressor Flow Modeling," Final Report for Naval Air Systems Command contract no. N00014-84-C-0354, July, AD-B102 101. 
Davis, R. L., Hobbs, D. E., and Weingold, H. D., 1988, "Prediction of Compressor Cascade Performance Using a Navier-Stokes Technique," ASME Paper 88-GT-96, International Gas Turbine Conference, Amsterdam, The Netherlands, June.

Gibeling, H. J., Weinberg, B. C., Shamroth, S. J, and McDonald, H., 1986, "Flow through a Compressor Stage," Report R86-910004-F, Scientific Research Associates, Inc., Glastonbury, Conn., May.

Gundy-Burlet, K. L., Rai, M. M., and Dring, R. P., 1989, "Two-Dimensional Computations of Multi-stage Compressor Flows Using a Zonal Approach," AIA A Paper 89-2452, Monterey, Calif., July.

Joslyn, H. D., and Dring, R. P., 1989a, "Multi-Stage Compressor Airfoil Aerodynamics: Part I - Airfoil Potential Flow Analysis," J. Propulsion Power, Vol. 5, No. 4, July August, pp. $457-462$.

Joslyn, H. D., and Dring, R. P., 1989b, "Multi- Stage Compressor Airfoil Aerodynamics: Part I - Airfoil Boundary-Layer Analysis," J. Propulsion Power, Vol. 5, No. 4, July - August, pp. 463 - 468.

Rai, M. M., and Chakravarthy, S. R., 1986, "An Implicit Form for the Osher Upwind Scheme," AIAA J., Vol.
24, No. 5, May, pp. 735-743.

Rai, M. M., 1987, "Navier-Stokes Simulations of Rotor/Stator Interactions Using Patched and Overlaid Grids," J. Propulsion Power, Vol. 3, No. 5, Sept., pp. 387-396.

Rai, M. M. and Madavan, N. K., 1988, "Multi-airfoil Navier-Stokes simulations of Turbine Rotor-Stator Interaction," AIA A Paper 88-0361, Reno, Nev., Jan.

Stauter, R. C., Dring, R. P., and Carta, F. O., 1990, "Temporally and Spatially Resolved Flow in a Two-Stage Axial Compressor, Part 1 - Experiment," to be presented at the 1990 ASME-IGTI Gas Turbine Conference in Brussels, Belgium.

Subramanian, S. V., Bozzola, R., and Povinelli, L. A., 1986, "Computation of Three-Dimensional, Rotational Flow Through Turbomachinery Blade Rows for Improved Aerodynamic Design Studies," ASME Paper 86-GT-26, International Gas Turbine Conference, Dusseldorf, West Germany, June.

Weinberg, B. C., Yang, R.-J., McDonald, H., and Shamroth, S. J., 1986, "Calculations of Two- and ThreeDimensional Transonic Cascade Flow Fields Using the Navier-Stokes Equations," J. Eng. Gas Turbines and Power, Vol. 108, Jan., pp. 93-102. 


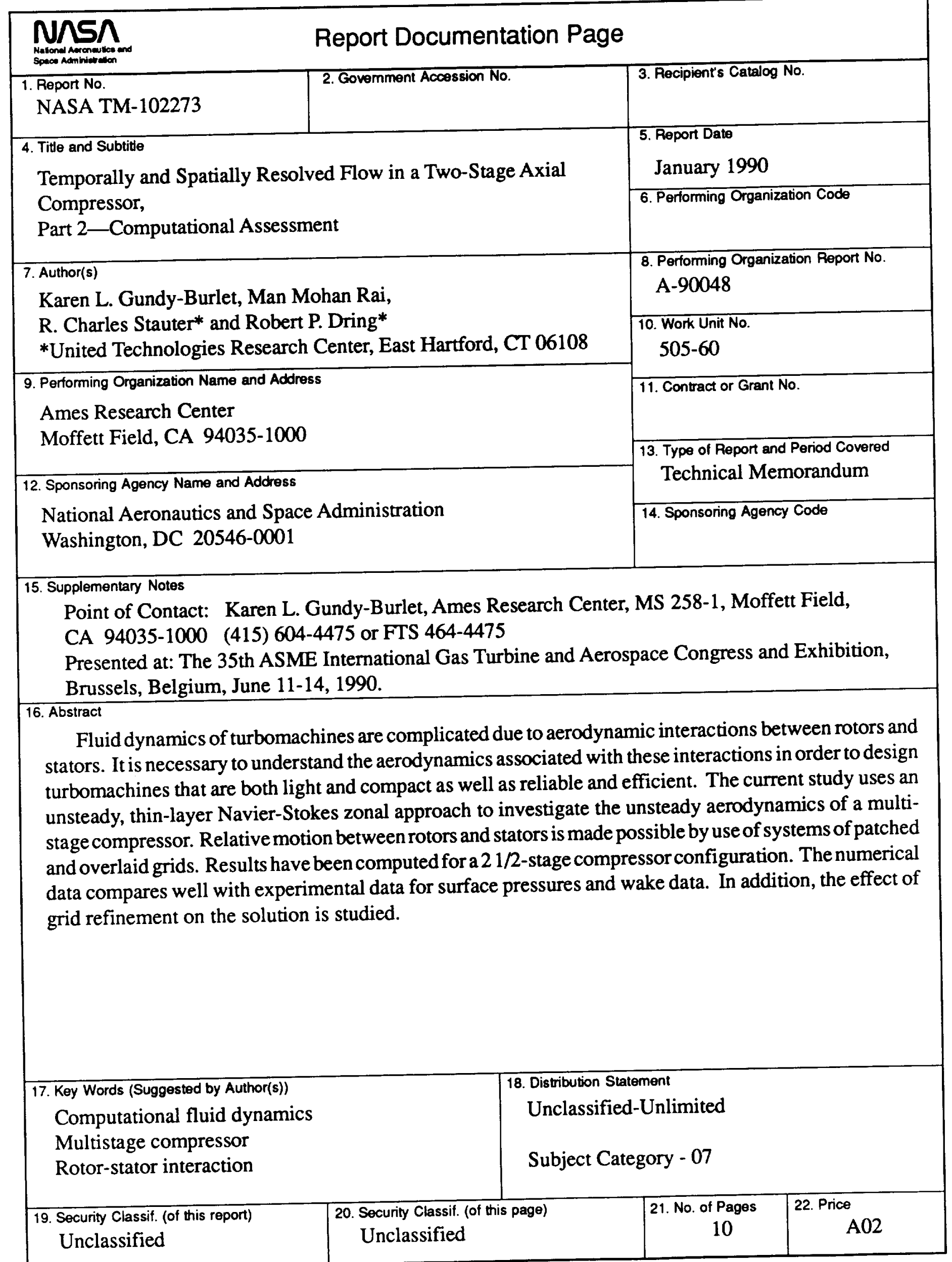




